\title{
EFFICIENT AND GREEN CATALYTIC SYNTHESIS OF DIHYDROPYRIMIDINONE (THIONE) DERIVATIVES USING COBALT NITRATE IN SOLVENT-FREE CONDITIONS
}

\author{
MASOUD NASR-ESFAHANI, * MORTEZA MONTAZEROZOHORI, MARYAM AGHEL-MIRREZAEE AND \\ HASSAN KASHI
}

Department of Chemistry, Yasouj University, Yasouj 75918-74831, Iran

(Received: May 14, 2013 - Accepted: December 14, 2013)

\begin{abstract}
A series of 3,4-dihydropyrimidin-2 $(1 \mathrm{H})$-one(thione) derivatives was synthesized using $\mathrm{Co}\left(\mathrm{NO}_{3}\right)_{2} \cdot 6 \mathrm{H}_{2} \mathrm{O}$ in solvent-free condition. Avoiding organic solvents during the chemical reactions leading to an economic approach is effective. The reaction is characterized by high efficiency, short reaction time, high yields, simple experimental procedure, availability of catalyst and environmentally friendly reaction conditions.
\end{abstract}

Keywords: Dihydropyrimidinone, Dihydropyrimidinthione, Cobalt(II)Nitrate, Solvent-free, One-pot synthesis

\section{INTRODUCTION}

Synthesis of 3,4-dihydropyrimidine-2(1H)-Ones (DHPMs) through onepot reaction of aromatic aldehyde, urea and ethyl acetoacetate in acid ethanol solution was initiated by Biginelli in $1893^{1}$. These compounds occupied an important place in medicinal and synthetic organic chemistry, mainly because of their wide range of biological activities ${ }^{2}$.

Notably, monastrol (1) is the only cell-permeable molecule that blocks normal bipolar spindle assembly in mammalian cells causing cell cycle arrest ${ }^{3}$, and is considered a lead for the development of new anticancer drugs ${ }^{4}$, while the appropriately functionalized DHPM analogs have emerged as orally active antihypertensive agents $(\mathbf{2}, \mathbf{4})^{5}$.

Many biological activities such as anticancer, antifungal, anti HIV have been exhibited using the representatives such as batzelladines, ptilomycalines (3) and crambescidines (Fig. 1) ${ }^{6}$.<smiles></smiles>

1<smiles>CC1=C(C(=O)O)C(c2cccc([N+](=O)[O-])c2)N(C(N)=O)C(=O)N1</smiles>

2
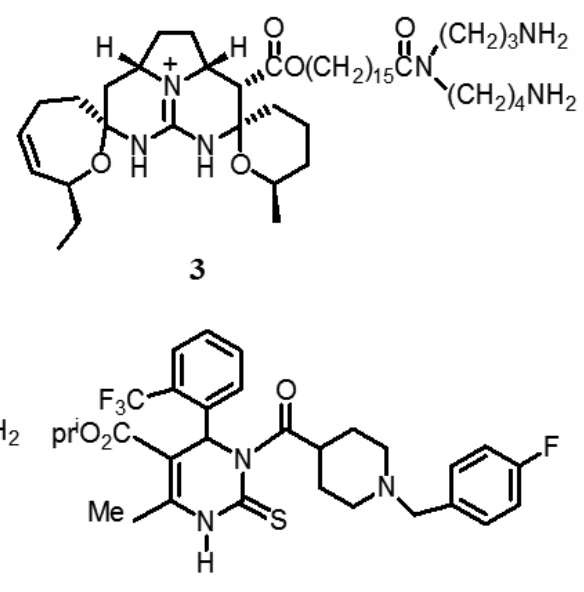

4
$<<$ Fig. $1>>$

The Biginelli method was developed ${ }^{7}$ and many catalysts such as $\mathrm{CaF}_{2}$ ${ }^{8}, \mathrm{Sr}(\mathrm{OTf})_{2}{ }^{9}, \mathrm{PPh}_{3}{ }^{10}$, tetrabutylammonium bromide (TBAB) ${ }^{11}$, copper(II) sulfamate ${ }^{12}$, phenyl phosphonic acid ${ }^{13}, \gamma-\mathrm{Fe}_{2} \mathrm{O}_{3} / \mathrm{CuO}$ (on the nanoscale) ${ }^{14}$ and so on have been used in the Biginelli reaction. Also microwave irradiation ${ }^{15}$, ultrasound irradiation ${ }^{16}$ and/or ionic liquids ${ }^{17}$ have been used. However, many of these methods suffer from drawbacks such as the use of expensive reagents, strong acidic conditions, long reaction times and use of expensive and poisonous solvents. Therefore, the introduction of a more efficient and milder methods accompanied with higher yields are in demand yet. In recent years with the development of green chemistry technology, multi components reactions under solvent-free conditions with a solid catalyst are considered as an important subject ${ }^{18}$. Solid acids have emerged as potential alternate catalysts to the common liquid acids due to their safe natures, enhanced selectivity, requirements in catalytic amounts and easier work up ${ }^{19}$.

Solid catalysts are harmless to the environment due to safety, no corrosion and reduction of the amount of waste residuals. So many reactions in organic chemistry of the solid catalyst are used. Following our interest in producing 3,4-dihydropyrimidine- $2(1 \mathrm{H})$-ones ${ }^{20}$, a study to revisit this reaction in a parallel combinatorial fashion using $\mathrm{Co}\left(\mathrm{NO}_{3}\right)_{2} \cdot 6 \mathrm{H}_{2} \mathrm{O}$ in a solvent-free synthesis approach was initiated.

\section{EXPERIMENTAL}

Chemicals were purchased from Merck, Fluka and Aldrich chemical companies. Melting points were determined using a Barnstead/Electothermal (BI) capillary apparatus and are uncorrected. IR spectra were recorded from $\mathrm{KBr}$ discs on a JASCO FT-IR-680. ${ }^{1} \mathrm{H}$ NMR spectra were recorded with Brucker ultrasheilld NMR 400 machines. NMR spectra were obtained on solution in DMSO- $\mathrm{d}_{6}$ using TMS as internal standard.

General procedure for the synthesis of 3,4-dihydropyrimidin-2(1H)ones(thiones)

A mixture of aldehyde $(1.0 \mathrm{mmol}), \quad \beta$-dicarbonyl $(1.0 \mathrm{mmol})$, $\mathrm{Co}\left(\mathrm{NO}_{3}\right)_{2} \cdot 6 \mathrm{H}_{2} \mathrm{O}(0.15 \mathrm{mmol})$ and urea or thiourea $(1.5 \mathrm{mmol})$ was magnetically stirred at $80{ }^{\circ} \mathrm{C}$ in solvent-free condition. After completion of the reaction, as indicated by TLC (EtOAc/n-hexane, 1:4), the reaction mixture was filtered and the residue recrystallized from ethanol to afford the pure product. All products were characterized by $\mathrm{mp}$, IR and ${ }^{1} \mathrm{H}$ NMR spectra. The physical and spectroscopic data of new compounds is given bellow:

Methyl 4-(2,6-dichlorophenyl)-6-methyl-2-oxo-1,2,3,4 tetrahydropyrimidine-5-carboxylate (8q): $\mathrm{Mp}: 292-294{ }^{\circ} \mathrm{C} ; \mathrm{R}_{f}=0.43$ (n-hexane:ethyl acetate $=4: 1) ; \operatorname{IR~}\left(\overline{\mathbf{v}}, \mathrm{cm}^{-1}\right): 3320,3227,2976,1698,1652,1661,1577,1497$, 1284, 1236; ${ }^{1} \mathrm{H}$ NMR (400 MHz, DMSO-d $) \delta(\mathrm{ppm}): 2.06$ (s, 3H), 2.19 (s, $3 \mathrm{H}), 6.18(\mathrm{~s}, 1 \mathrm{H}), 7.26(\mathrm{t}, 1 \mathrm{H}, J=7.6 \mathrm{~Hz}), 7.40(\mathrm{~d}, 2 \mathrm{H}, J=8.0 \mathrm{~Hz}), 7.62(\mathrm{~s}$, $1 \mathrm{H}), 9.25(\mathrm{~s}, 1 \mathrm{H})$; Anal. Calcd. for $\mathrm{C}_{13} \mathrm{H}_{12} \mathrm{Cl}_{2} \mathrm{~N}_{2} \mathrm{O}_{3}: \mathrm{C}, 49.54 ; \mathrm{H}, 3.84 ; \mathrm{Cl}, 22.50$; N, 8.89; O, 15.23; found: C 49.45, H 3.90, N 8.80.

Methyl 4-(2-bromophenyl)-6-methyl-2-oxo-1,2,3,4-tetrahydropyrimidine-5-carboxylate (8r): Mp: $249-251^{\circ} \mathrm{C} ; \mathrm{R}_{f}=0.33$ ( $n$-hexane:ethyl acetate $=$ 4:1); IR $\left(\overline{\mathbf{v}}, \mathrm{cm}^{-1}\right): 3363,3249,2929,1703,1623,1458,1381,1229 ;{ }^{1} \mathrm{H}$ NMR $(400 \mathrm{MHz}$, DMSO-d $) \delta(\mathrm{ppm}): 2.05(\mathrm{~s}, 3 \mathrm{H}), 2.34(\mathrm{~s}, 3 \mathrm{H}), 5.63(\mathrm{~s}, 1 \mathrm{H}), 7.20$ $(\mathrm{t}, 1 \mathrm{H}, J=6.0 \mathrm{~Hz}), 7.26(\mathrm{~d}, 1 \mathrm{H}, J=6.4 \mathrm{~Hz}), 7.37(\mathrm{t}, 1 \mathrm{H}, J=7.2 \mathrm{~Hz}), 7.61(\mathrm{~d}$, $1 \mathrm{H}, J=7.2 \mathrm{~Hz}), 7.71(\mathrm{~s}, 1 \mathrm{H}), 9.30(\mathrm{~s}, 1 \mathrm{H})$; Anal. Calcd. for $\mathrm{C}_{13} \mathrm{H}_{13} \mathrm{BrN}_{2} \mathrm{O}_{3}$ : C, 48.02; H, 4.03; Br, 24.57; N, 8.62; O, 14.76; found: C 47.93, H 4.10, N 8.54.

Methyl 4-(2,4-dichlorophenyl)-6-methyl-2-thioxo-1,2,3,4-tetrahydropyrimidine-5-carboxylate (8y): Mp: $214-215{ }^{\circ} \mathrm{C} ; \mathrm{R}_{f}=0.50$ ( $n$-hexane:ethyl acetate $=4: 1) ; \operatorname{IR}\left(\overline{\mathbf{v}}, \mathrm{cm}^{-1}\right): 3406,3179,2949,1678,1652,1563,1464,1203$; ${ }^{1} \mathrm{H}$ NMR (400 MHz, DMSO-d $)$ ) $\delta(\mathrm{ppm}): 2.30(\mathrm{~s}, 3 \mathrm{H}), 3.34(\mathrm{~s}, 3 \mathrm{H}), 5.58(\mathrm{~s}$, $1 \mathrm{H}), 7.28(\mathrm{~d}, 1 \mathrm{H}, J=8.8 \mathrm{~Hz}), 7.43(\mathrm{~d}, 1 \mathrm{H}, J=8.0 \mathrm{~Hz}), 7.58(\mathrm{~s}, 1 \mathrm{H}, J=7.2$ $\mathrm{Hz}), 9.65(\mathrm{~s}, 1 \mathrm{H}), 10.45(\mathrm{~s}, 1 \mathrm{H})$; Anal. Calcd. for $\mathrm{C}_{13} \mathrm{H}_{12} \mathrm{Cl}_{2} \mathrm{~N}_{2} \mathrm{O}_{2} \mathrm{~S}: \mathrm{C}, 47.14$; $\mathrm{H}, 3.65 ; \mathrm{Cl}, 21.41 ; \mathrm{N}, 8.46$; O, 9.66; S, 9.68; found: $\mathrm{C}_{13} 47.03$, H 3.72, N 8.39.

Methyl 4-(3-chlorophenyl)-6-methyl-2-thioxo-1,2,3,4-tetrahydropyrimidine-5-carboxylate (8z): Mp: $248-249{ }^{\circ} \mathrm{C} ; \mathrm{R}=0.35$ ( $n$-hexane:ethyl acetate $=4: 1) ; \operatorname{IR~}\left(\overline{\mathbf{v}}, \mathrm{cm}^{-1}\right): 3316,3176,2968,1678,1662,1574,1434,1385$, 
1285; ${ }^{1} \mathrm{H}$ NMR (400 MHz, DMSO-d $) \delta(\mathrm{ppm}): 2.29$ (s, 3H), 3.56 (s, 3H), 5.17 $(\mathrm{s}, 1 \mathrm{H}), 7.17-7.36(\mathrm{~m}, 4 \mathrm{H}), 9.71(\mathrm{~s}, 1 \mathrm{H}), 10.45(\mathrm{~s}, 1 \mathrm{H})$; Anal. Calcd. for $\mathrm{C}_{13} \mathrm{H}-$ $\mathrm{ClN}_{2} \mathrm{O}_{2} \mathrm{~S}: \mathrm{C}, 52.61 ; \mathrm{H}, 4.42 ; \mathrm{Cl}, 11.95 ; \mathrm{N}, 9.44 ; \mathrm{O}, 10.78 ; \mathrm{S}, 10.80$; found: $\mathrm{C}$ $52.53, \mathrm{H} 4.50, \mathrm{~N} 9.37$.

\section{RESULTS AND DISCUSSION}

In this paper, we report synthesis of the dihydropyrimidinones and dihydropyrimidinthiones using cobalt nitrate hexahydrate as catalyst at $80{ }^{\circ} \mathrm{C}$ in solvent-free condition (Scheme 1).
$\mathrm{R}^{1} \mathrm{CHO}$<smiles>[R]C(=O)CC(C)=O</smiles>

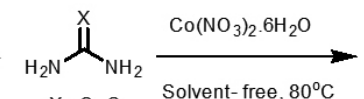
6a-c

$5 a-x$ 7a-b<smiles>[R7]C(=O)C1=C(C)NC(=[V])NC1[R1]</smiles>
8a-x
Scheme 1. Synthesis of DHPMs 8a-x.

Effect of catalyst concentration and solvent

The catalyst concentration was varied over a range of $5-25 \mathrm{~mol} \%$ of $\mathrm{Co}\left(\mathrm{NO}_{3}\right)_{2} \cdot 6 \mathrm{H}_{2} \mathrm{O}$ on the basis of the total volume of the reaction mixture. Table 1 shows the effect of catalyst concentration on the reaction of benzaldehyde, ethylacetoacetate and urea. The yield of the corresponding dihydropyrimidinone increased with increasing catalyst concentration from 5 to $15 \mathrm{~mol} \%$. Further addition of catalyst had no noticeable effect on the yield. This was due to over an obvious concentration, there have an excess of catalyst sites beyond what is actually required by the reactant substrates, and the additional catalyst does not increase the rate of the reaction. Therefore, in all further reactions $15 \mathrm{~mol} \%$ were used.

Table 1. Investigation of catalyst effects in the synthesis of 5-(Ethoxycarbonyl)-6-methyl-4-phenyl-3,4-dihydropyrimidin-2(1H)-one under solvent-free conditions ${ }^{\mathrm{a}}$

\begin{tabular}{|c|c|c|c|}
\hline Entry & $\begin{array}{c}\text { Amount of catalyst } \\
(\mathrm{mol} \%)\end{array}$ & Time (min) & Yields (\%) \\
\hline 1 & 5 & 80 & 85 \\
\hline 2 & 10 & 65 & 90 \\
\hline 3 & 15 & 40 & 93 \\
\hline 4 & 20 & 40 & 94 \\
\hline 5 & 25 & 50 & 94 \\
\hline
\end{tabular}

bIsolated yields

Then, the solvent effect in the condensation of benzaldehyde ( $1 \mathrm{mmol})$, urea $(1.5 \mathrm{mmol})$ and ethyl acetoacetate $(1 \mathrm{mmol})$ in the presence of $\mathrm{Co}\left(\mathrm{NO}_{3}\right)_{2} \cdot 6 \mathrm{H}_{2} \mathrm{O}$ $(0.15 \mathrm{mmol})$ as a model has been studied. As shown in Table 2, among the tested solvents, such as ethanol, methanol, acetonitrile, water, chloroform and a solvent-free system, the best result was obtained after 40 min under solventfree conditions in excellent yield (90\%).

Table 2. Solvent effect on the reaction of benzaldehyde, urea and ethyl acetoacetate catalyzed by $\mathrm{Co}\left(\mathrm{NO}_{3}\right)_{2} \cdot 6 \mathrm{H}_{2} \mathrm{O}$.

\begin{tabular}{|c|c|c|c|}
\hline Entry & Solvent (reflux) & Time(h) & Yielda) $^{\text {(\%) }}$ \\
\hline 1 & $\mathrm{CH}_{3} \mathrm{OH}$ & 20 & 68 \\
\hline 2 & $\mathrm{CH}_{3} \mathrm{CH}_{2} \mathrm{OH}$ & 20 & 70 \\
\hline 3 & $\mathrm{CH}_{3} \mathrm{CN}$ & 20 & 60 \\
\hline 4 & $\mathrm{CH}_{3} \mathrm{Cl}$ & 20 & 55 \\
\hline 5 & $\mathrm{H}_{2} \mathrm{O}$ & 20 & 65 \\
\hline 6 & Solvent-free $^{\mathrm{b}}$ & 0.6 & 90 \\
\hline
\end{tabular}

a Isolated yields

${ }^{\mathrm{b}}$ At $80{ }^{\circ} \mathrm{C}$

We began our studies with the reaction of benzaldehyde (5a), ethylacetoacetate (6a) and urea (7a) as a model reaction. For this purpose, various parameters such as molar ratio of reactants, catalyst and reaction temperature were optimized. The results showed that use of $1 \mathrm{mmol}$ benzaldehyde, $1 \mathrm{mmol}$ ethylacetoacetate, $1.5 \mathrm{mmol}$ of urea or thiourea and $1.5 \mathrm{mmol}$ catalyst under solvent-free condition at $80{ }^{\circ} \mathrm{C}$, the product of dihydropyrimidinone (thiones) with $90 \%$ efficiency was achieved. Then under this condition, dihydropyrimidinones and dihydropyrimidinthiones by various aromatic and aliphatic aldehydes were synthesized. The results were presented in table 3. As shown, various aromatic aldehydes bearing either electron-releasing or electron-withdrawing substituents can lead to high yields. The use of methyl acetoacetate or acetylacetone as 1,3-dicarbonyl moiety in place of ethyl acetoacetate also gave similar results (Table 3, entries 16-21, 25-27). Furthermore, substrate with two formyl group (entry 10) was produced the corresponding bis-dihydropyrimidinone in short time and excellent yield (Scheme 2).

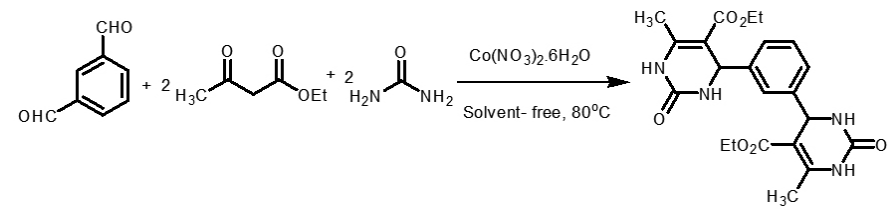

Scheme 2. Synthesis of ethyl-4-(3-(5-(ethoxycarbonyl)-1,2,3,4tetrahydro-6-methyl-2-oxopyrimidine-4-yl)-Phenyl)-1,2,3,4-tetrahydro-6methyl-2-oxopyrimidine-5-carboxylate.

The reactions of acid-sensitive substrates such as 2-thiophenecarbaldehyde and cinnamaldehyde also proceeded well to give the dihydropyrimidinone without any side products (entry 11 and 12). However, aliphatic aldehydes such as butanal, as observed previously, reacted over longer times with a reduced yield (entry 13, 80 min time, $35 \%$ yield) compared with the aromatic compounds under our reaction conditions ${ }^{20}$.

To use of $\mathrm{Co}\left(\mathrm{NO}_{3}\right)_{2} \cdot 6 \mathrm{H}_{2} \mathrm{O}$ in large scale synthesis especially in chemical laboratory, a typical reaction was performed for synthesis of $\mathbf{8 a}$ with tenfold amounts of reactants and catalyst with respect to one mentioned in the experimental section. The result showed that the yield of $87 \%$ in these conditions that is comparable with one in table 3 .

Three possible mechanisms are proposed for Biginelli reaction according to the literature but generally accepted reaction mechanism includes the acidcatalyzed formation of $\mathrm{C}-\mathrm{N}$ bond from the benzaldehyde and urea (pathway A, Scheme 3) ${ }^{36}$. According to reported results the pathway A is characteristic for the Brønsted type of catalysts whereas Lewis acid type of catalysts follows the pathway B (ureido-crotonate mechanism) ${ }^{37}$. For clarifying the role of the catalyst, three separated reactions were performed (pathways A-C, Scheme 3 ) under the optimized reaction conditions ( $15 \mathrm{~mol} \%$ of cobalt(II) nitrate at $80^{\circ} \mathrm{C}$ in solvent.free condition and reflux temperature in acetonitrile during $30 \mathrm{~min}$ and $20 \mathrm{~h}$, respectively). The prolonged heating of benzaldebyde and ethyl acetoacetate (pathway C) or benzaldehyde and urea (pathway A) did not undergo the expected reactions to yield of products whereas the reaction of ethyl acetoacetate and urea furnished the ureido-crotonate (pathway B). These observations clearly indicate that Biginelli reaction catalyzed by cobalt(II) nitrate proceeds predominately.

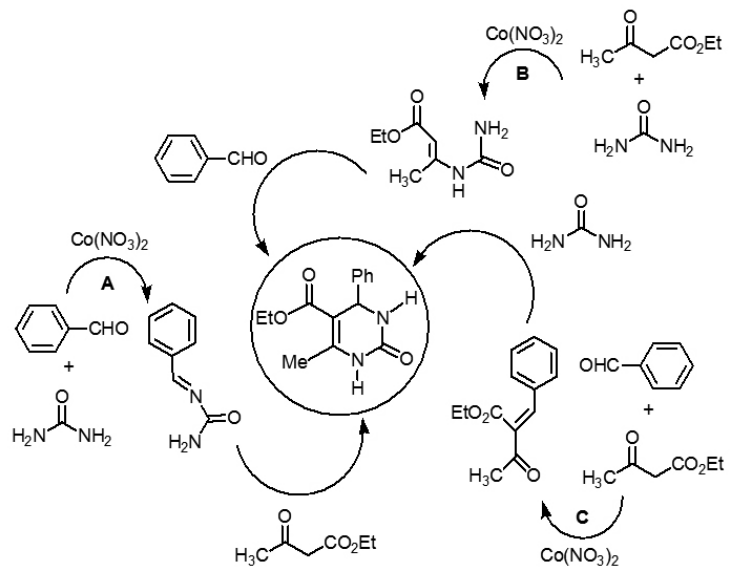

Scheme 3. Three proposed possible mechanisms for Biginelli reaction. 
Comparative results

In order to show the ability of our method with respect to previous reports, our result for synthesis of 5-(ethoxycarbonyl)-6-methyl-4-phenyl-3,4dihydropyrimidin-2 $(1 \mathrm{H})$-ones $(\mathbf{8 a})$ in comparison to other methods for preparation of this compound have been summarized in table 4 . As shown in table 4 , the yield/time ratio of the present method is better or comparable with the other reported results.

Table 3. Synthesis of 3,4-dihydropyrimidin-2-(1H)-ones(thiones) Catalyzed by $\mathrm{Co}\left(\mathrm{NO}_{3}\right)_{2} \cdot 6 \mathrm{H}_{2} \mathrm{O}$ under solvent-free Conditions ${ }^{\mathrm{a}}$

\begin{tabular}{|c|c|c|c|c|c|c|c|c|}
\hline entry & $\mathrm{R}^{1}$ & $\mathrm{R}^{2}$ & $\mathrm{X}$ & Product & Time (min) & Yield $(\%)^{b}$ & $\mathrm{Mp}\left({ }^{\circ} \mathrm{C}\right)$ & Ref \\
\hline 1 & $\mathrm{C}_{6} \mathrm{H}_{5}$ & OEt & $\mathrm{O}$ & $8 \mathbf{a}$ & 40 & 90 & 208-209 & 21 \\
\hline 2 & $4-\mathrm{CH}_{3} \mathrm{OC}_{6} \mathrm{H}_{4}$ & $\mathrm{OEt}$ & $\mathrm{O}$ & $8 \mathbf{b}$ & 40 & 92 & 205-207 & 13 \\
\hline 3 & 4- $\mathrm{CH}_{3} \mathrm{C}_{6} \mathrm{H}_{4}$ & OEt & $\mathrm{O}$ & $8 c$ & 50 & 90 & $215-217$ & 13 \\
\hline 4 & $3-\mathrm{BrC}_{6} \mathrm{H}_{4}$ & OEt & $\mathrm{O}$ & $8 d$ & 65 & 79 & $185-187$ & 22 \\
\hline 5 & $4-\mathrm{ClC}_{6} \mathrm{H}_{4}$ & OEt & $\mathrm{O}$ & $8 \mathrm{e}$ & 65 & 80 & $212-214$ & 13 \\
\hline 6 & $2-\mathrm{CH}_{3} \mathrm{OC}_{6} \mathrm{H}_{4}$ & OEt & $\mathrm{O}$ & $8 f$ & 55 & 85 & $257-259$ & 24 \\
\hline 7 & $2-\mathrm{ClC}_{6} \mathrm{H}_{4}$ & OEt & $\mathrm{O}$ & $8 \mathrm{~g}$ & 70 & 85 & 216-218 & 13 \\
\hline 8 & $4-\mathrm{NO}_{2} \mathrm{C}_{6} \mathrm{H}_{4}$ & $\mathrm{OEt}$ & $\mathrm{O}$ & $8 \mathrm{~h}$ & 75 & 80 & 207-209 & 13 \\
\hline 9 & $2-\mathrm{NO}_{2} \mathrm{C}_{6} \mathrm{H}_{4}$ & OEt & $\mathrm{O}$ & $8 \mathbf{i}$ & 35 & 88 & 208-209 & 25 \\
\hline 10 & $3-\mathrm{CHOC}_{6} \mathrm{H}_{4}$ & OEt & $\mathrm{O}$ & $8 \mathrm{j}$ & 24 & 90 & $212-214$ & 26 \\
\hline 11 & 2-Thienyl & OEt & $\mathrm{O}$ & $8 k$ & 65 & 85 & $213-215$ & 23 \\
\hline 12 & $\mathrm{C}_{6} \mathrm{H}_{5}-\mathrm{CH}=\mathrm{CH}$ & OEt & $\mathrm{O}$ & 81 & 40 & 75 & $230-232$ & 27 \\
\hline 13 & $n$-Pr & OEt & $\mathrm{O}$ & $8 m$ & 80 & 35 & $178-180$ & 23 \\
\hline 14 & $2,4-\mathrm{Cl}_{2} \mathrm{C}_{6} \mathrm{H}_{3}$ & OEt & $\mathrm{O}$ & $8 n$ & 50 & 80 & $251-252$ & 22 \\
\hline 15 & $3-\mathrm{NO}_{2} \mathrm{C}_{6} \mathrm{H}_{4}$ & OEt & $\mathrm{O}$ & 80 & 14 & 93 & $227-228$ & 28 \\
\hline 16 & $\mathrm{C}_{6} \mathrm{H}_{5}$ & $\mathrm{OMe}$ & $\mathrm{O}$ & $8 p$ & 40 & 88 & 191-193 & 29 \\
\hline 17 & $2,6-\mathrm{Cl}_{2} \mathrm{C}_{6} \mathrm{H}_{3}$ & $\mathrm{OMe}$ & $\mathrm{O}$ & $8 q$ & 45 & 80 & 292-294 & - \\
\hline 18 & $2-\mathrm{BrC}_{6} \mathrm{H}_{4}$ & $\mathrm{OMe}$ & $\mathrm{O}$ & $8 r$ & 50 & 85 & 249-251 & - \\
\hline 19 & $\mathrm{C}_{6} \mathrm{H}_{5}$ & $\mathrm{Me}$ & $\mathrm{O}$ & $8 s$ & 45 & 90 & $232-234$ & 30 \\
\hline 20 & $4-\mathrm{CH}_{3} \mathrm{C}_{6} \mathrm{H}_{4}$ & $\mathrm{Me}$ & $\mathrm{O}$ & $8 t$ & 45 & 85 & $250-253$ & 31 \\
\hline 21 & $4-\mathrm{CH}_{3} \mathrm{OC}_{6} \mathrm{H}_{4}$ & $\mathrm{Me}$ & $\mathrm{O}$ & $8 u$ & 50 & 90 & 171-169 & 30 \\
\hline 22 & 4- $\mathrm{CH}_{3} \mathrm{OC}_{6} \mathrm{H}_{4}$ & OEt & $\mathrm{S}$ & $8 v$ & 70 & 90 & 191-194 & 32 \\
\hline 23 & $4-\mathrm{HOC}_{6} \mathrm{H}_{4}$ & OEt & $\mathrm{S}$ & $8 w$ & 80 & 85 & 197-199 & 33 \\
\hline 24 & $2-\mathrm{CH}_{3} \mathrm{OC}_{6} \mathrm{H}_{4}$ & $\mathrm{OEt}$ & $\mathrm{S}$ & $8 x$ & 30 & 90 & $200-202$ & 34 \\
\hline 25 & $2,4-\mathrm{Cl}_{2} \mathrm{C}_{6} \mathrm{H}_{3}$ & $\mathrm{OMe}$ & $\mathrm{S}$ & $8 y$ & 55 & 90 & $254-255$ & - \\
\hline 26 & $3-\mathrm{ClC}_{6} \mathrm{H}_{4}$ & $\mathrm{OMe}$ & $\mathrm{S}$ & $8 z$ & 60 & 84 & $248-249$ & - \\
\hline 27 & $4-\mathrm{ClC}_{6} \mathrm{H}_{4}$ & $\mathrm{OMe}$ & $\mathrm{S}$ & $8 a^{\prime}$ & 60 & 85 & $241-243$ & 35 \\
\hline
\end{tabular}

${ }^{a}$ Reaction conditions: aldehyde ( $\left.1 \mathrm{mmol}\right), \beta$-dicarbonyl $(1 \mathrm{mmol})$, urea or thiourea $(1.5 \mathrm{mmol})$,

$\mathrm{Co}\left(\mathrm{NO}_{3}\right)_{2} \cdot 6 \mathrm{H}_{2} \mathrm{O}(0.15 \mathrm{mmol}), 80^{\circ} \mathrm{C} ;{ }^{\mathrm{b}}$ Isolated yield

Table 4. Comparison of efficiency of various catalysts in synthesis of 5-(Ethoxycarbonyl)-6-methyl-4-phenyl-3,4-dihydropyrimidin-2(1H)-ones

\begin{tabular}{|c|c|c|c|c|c|}
\hline Entry & Catalyst & Amount of catalyst $\mathrm{t}^{\mathrm{b}}$ & Conditions & Yield/Time $^{\text {a }}$ & Ref \\
\hline 1 & $\mathrm{Sr}(\mathrm{OTf})_{2}$ & 5 & Solvent-free $/ 70^{\circ} \mathrm{C}$ & $97 / 4$ & 38 \\
\hline 2 & {$\left[\mathrm{Al}\left(\mathrm{H}_{2} \mathrm{O}\right)_{6}\right]\left(\mathrm{BF}_{4}\right)_{3}$} & 10 & $\mathrm{CH}_{3} \mathrm{CN}$ (reflux) & $81 / 20$ & 39 \\
\hline 3 & $\mathrm{Zr}\left(\mathrm{H}_{2} \mathrm{PO}_{4}\right)_{2}$ & 7 & Solvent-free $/ 90{ }^{\circ} \mathrm{C}$ & $88 / 1$ & 40 \\
\hline 4 & $\mathrm{LiBr}$ & 10 & $\mathrm{CH}_{3} \mathrm{CN}$ (reflux) & $92 / 3$ & 41 \\
\hline 5 & $\mathrm{SbCl}_{3}$ & 100 & $\mathrm{CH}_{3} \mathrm{CN}$ (reflux) & $90 / 18$ & 42 \\
\hline 6 & $\mathrm{CaF}_{2}$ & 10 & $\mathrm{C}_{2} \mathrm{H}_{5} \mathrm{OH} /$ (reflux) & $98 / 2$ & 43 \\
\hline 7 & Chloroacetic acid & 10 & Solvent-free $/ 90^{\circ} \mathrm{C}$ & $92 / 3$ & 44 \\
\hline 8 & $\mathrm{NH}_{4} \mathrm{Cl}$ & 40 & Solvent-free $/ 100{ }^{\circ} \mathrm{C}$ & $90 / 3$ & 45 \\
\hline 9 & Triphenylphosphine & 10 & Solvent-free $/ 100{ }^{\circ} \mathrm{C}$ & $70 / 10$ & 46 \\
\hline 10 & $\mathrm{TiCl}_{4}-\mathrm{MgCl}_{2}$ & 10 & Solvent-free $/ 100{ }^{\circ} \mathrm{C}$ & $90 / 3$ & 47 \\
\hline 11 & $\mathrm{Co}\left(\mathrm{NO}_{3}\right)_{2} \cdot 6 \mathrm{H}_{2} \mathrm{O}$ & 15 & Solvent free $/ 80{ }^{\circ} \mathrm{C}$ & $90 / 0.6$ & - \\
\hline
\end{tabular}

a Values refer to yield(\%)/time(h)

${ }^{\mathrm{b}}$ Amount of catalysts are in $\mathrm{mol} \%$ 


\section{CONCLUSIONS}

In summary, we have described an improved procedure for the synthesis of dihydropyrimidinones and dihydropyrimidinthiones using cobalt(II) nitrate hexahydrate as heterogeneous catalyst. For clarifying the role of the catalyst, three separated reactions were performed that the results clearly indicated that the reaction catalyzed by $\mathrm{Co}\left(\mathrm{NO}_{3}\right)_{2}$ proceeds predominately through ureidocrotonate intermediate, which this achievement well supports the necessity of Lewis type catalyst for the Biginelli reaction. The mild reaction conditions, rapid conversion, high yields, simple experimental procedure, availability of catalyst are some notable advantages of the present method. Moreover, compatibility with the environment, more efficiency and easy separation after synthesis are considered as another advantages of this catalyst loading. Most importantly, absence of organic solvents in this method contributes it to the development of green technology.

\section{ACKNOWLEDGMENTS}

We are grateful to the Yasouj University for financial assistance.

\section{REFERENCES}

1. P. Biginelli, Gazz. chim. Ital. 23, 360 (1893).

2. (a) C.O. Kappe, Acc. Chem. Res. 33, 879 (2000); (b) C.O. Kappe, Eur. J. Med. Chem. 35, 1043 (2000); (c) C.O. Kappe, Tetrahedron. 49, 6937 (1993).

3. (a) M. Gartner, N. Sunder-Plassmann, J. Seiler, M. Utz, I. Vernos, T. Surrey, A. Giannis, 6, 1173 (2005); (b) T.U. Mayer, T.M. Kapoor, T.J. Mitchison, S. Schreiber, Chem. Biol. 7, 275 (2000).

4. (a) T.M. Mayer, T.M. Kapoor, S.J. Haggarty, R.W. King, S.L. Schreiber, T.J. Mitchison, Science 286, 971 (1999). (b) C.O. Kappe, O.V. Shishkin, G. Uray, P. Verdino, Tetrahedron 56, 1859-1862 (2000).

5. (a) G.C. Rovnyak, K.S. Atwal, A. Hedberg, S.D. Kimball, S. Moreland, J.Z. Gougoutas, B. C. O'Reilly, J. Schwartz, M.F. Malley, J. Med. Chem. 35, 3254 (1992); (b) G.J. Grover, S. Dzwonczyk, D.M. McMullen, D.E. Normandin, C.S. Parham, P.G. Sleph, S. Moreland, J. Cardiovasc. Pharmacol. 26, 289 (1995)

6. (a) L. Heys, C.G. Moore, P.J. Murphy, Chem. Soc. Rev. 29, 57 (2000); (b) C.A. Bewley, S. Ray, F. Cohen, S.K. Collins, L.E. Overmann, J. Nat. Prod. 67, 1319 (2004).

7. K.S. Atwal, B.C. O-Reilly, J.Z. Gougoutas, M.F. Malley, Heterocycles 26, 1189 (1987).

8. S. Chitra, K. Pandiarajan, Tetrahedron Lett. 50, 2222 (2009).

9. W.K. Su, J.J. Li, Z.G. Zheng, Y.C. Shen, Tetrahedron Lett. 46, 6037 (2005).

10. A. Debache, M. Amimour, A. Belfaitah, S. Rhouati, B. Carboni, Tetrahedron Lett. 49, 6119 (2008).

11. B. Ahmad, R.A. Khan, M. Keshari, Tetrahedron Lett. 50, 2889 (2009).

12. L. Chen-Jiang, J.D. Wang, Molecules 14, 763 (2009).

13. D.S. Ashok, M.P. Sanjeev, S.P. Jitendra, V.Y. Manjusha, J. Chem. Pharm. Res. 3, 649 (2011).

14. D. Prodius, F. Macaer, V. Mereacre, S. Shova, Y. Lutsenco, Y.A. Simonov, C. Turta, Inorg. Chem. Commun. 12, 642 (2009).
15. B.K. Banik, A.T. Reddy, A. Datta, C. Mukhopadhyay, Tetrahedron Lett. 48, 7392 (2007).

16. Q. Wang, W. Pei, J. Iran. Chem. Soc. 7, 318 (2010).

17. J.J. Peng, Y.Q. Deng, Tetrahedron Lett. 42, 5917 (2001).

18. Y. Wang, J. Yu, H. Yang, Z. Miao, R. Chen, Lett. Org. Chem. 8, 264 (2011).

19. J.H. Clark, Acc. Chem. Res. 35, 791 (2002).

20. (a) M. Nasr-Esfahani, T. Abdizadeh, Phosphorus Sulfur 188, 596 (2013); (b) M. Nasr-Esfahani, S.J. Hoseini, F. Mohammadi, Chin. J. Catal. 32, 1484 (2011); (c) M. Nasr-Esfahani, B. Karami, M. Montazerozohori, K. Abdi, J. Heterocycl. Chem. 45, 1183 (2008); (d) M. Nasr-Esfahani, A.R. Khosropour, Bull. Korean Chem. Soc. 26, 1331 (2005).

21. Q. Sun, Y. Wang, Z. Ge, T. Cheng, R. Li, Synthesis 7, 1047 (2004).

22. N.K Hitendra, S. Manisha, M.P. Kaushik, Molecules. 12, 1341 (2007).

23. M. Muchchintala, H.O. Sang, K. Ketack, Y.D. Jung, Bull. Korean Chem. Soc. 29, 1752 (2008).

24. F.S Falsone, C.O. Kappe, Arkivoc 2, 122 (2001).

25. P. Shanmugam, P.T. Perumal, Tetrahedron 62, 9726 (2006)

26. S. Ko, C.F. Yao, Tetrahedron 62, 7293 (2006).

27. N.Y Fu, Y.F. Yuan, Z. Cao, S.W. Wang, J.T. Wang, C. Peppe, Tetrahedron 58, 4801 (2002).

28. T.S. Jin, H.X. Wang, C.Y. Xing, X.L. Li, T.S. Li, Synth. Commun. 34, 3009 (2004).

29. Y. Ma, C. Qian, L. Wang, M. Yang, J. Org. Chem. 65, 3864 (2000).

30. A. Debache, M. Amimour, A. Belfaitah, S. Rhouati, B. Carboni, Tetrahedron Lett. 49, 6119 (2008).

31. C.O. Kappe, O.V. Shishkin, G. Uray, P. Verdino, Tetrahedron 56, 1859 (2000).

32. A. Shaabani, A. Bazgir, F. Teimouri, Tetrahedron Lett. 44, 857 (2003).

33. A. Borse, M. Patil, N. Patil, R. Shinde, ISRN Org. Chem. Article ID 415645, 1 (2012)

34. C. Ramalingan, Y.W. Kwak, Tetrahedron 64, 5023 (2008).

35. S. Kumar, P. Sharma, K.K. Kapoor, M.S. Hundal, Tetrahedron 64, 536 (2008).

36. (a) F. Sweet, J. D. Fissekis, J. Am. Chem. Soc. 95, 8741 (1973); (b) C. O. Kappe, A, J. Org. Chem. 62, 7201 (1997).

37. I. Cepanec, M. Litvic, M. Filipan-Litvic, I. Grungold, Tetrahedron 63, 11822 (2007)

38. W.K. Su, J.J. Li, Z.G. Zheng, Y.C. Shen, Tetrahedron Lett. 46, 6037 (2005).

39. M. Litvic, I. Vecenaj, Z.M. Ladisic, M. Lovric, V. Vinkovic, M. FilipanLitvic, Tetrahedron 66, 3463 (2010).

40. S. Besoluk, M. Kukukislamoglu, M. Zengin, M. Arsalan, M. Nebioglu, Turk. J. Chem. 34, 411 (2010).

41. G. Maiti, P. Kundu, C. Guin, Tetrahedron Lett. 44, 2757 (2003).

42. I. Cepanec, M. Litvic, M. Filipan-Litvic, I. Grungold, Tetrahedron 63, 11822 (2007)

43. S. Chitra, K. Pandiarajan, Tetrahedron Lett. 50, 2222 (2009).

44. Y. Yu, D. Liu, C. Liu, G. Luo, Bioorg. Med. Chem. Lett. 17, 3508 (2007).

45. A. Shaabani, A. Bazgir, F. Teimouri, Tetrahedron Lett. 44, 857-859 (2003).

46. A. Debache, M. Amimour, A. Belfaitah, S. Rhouati, B. Carboni, Tetrahedron Lett. 49, 6119 (2008).

47. A. Kumar, R.A. Maurya, J. Mol. Catal. A: Chem. 272, 53 (2007). 